\title{
Editorial \\ Pharmacological approaches to the study of social behaviour
}

Behavioural Pharmacology 2015, 26:501-504

This Special Issue marks a new departure for Behavioural Pharmacology. Special Issues have always been a feature of this journal, appearing as a double issue every year since Volume 1 in 1990. This year, after dedicating a double Special Issue to the memory of Lex Cools (The behavioural pharmacology of the basal ganglia, Behavioural Pharmacology 26:1-2), the editors announced a second Special Issue showcasing new developments in the pharmacology of social behaviour, anticipating that we would receive sufficient papers to comfortably fill a single issue. We had not anticipated the overwhelming response that resulted in not a single, not a double, but a triple issue, which will be published as Volume 26, Issues 6, 7 and 8. The current issue (Part 1: Issue 26.6) contains eight review papers providing overviews of literature relating to social communication, models of autism, effects of drugs of abuse on social behaviour (and vice versa), and the pharmacology of sexual and maternal behaviour. Parts 2 (Issue 26.7) and 3 (Issue 26.8) will contain empirical studies of the two faces of sociopharmacology: social modulation of drug effects and drug effects on social behaviour. The papers to be included in Parts 2 and 3, and introduced below, are available online in the Behavioural Pharmacology 'published ahead of print' pipeline, which may be found at: http://journals.lww. com/behaviouralpharm/toc/publishahead.

\section{Part 1: Reviews}

The first review, by Wöhr et al., analyses the role of serotonin (5HT) in ultrasonic vocalizations (USVs), a key element of affective communication. The review covers isolation-induced distress USVs in mouse and rat pups, interaction-induced appetitive USVs in juvenile (during play) and adult (during sexual behaviour) rodents, and fear-induced aversive USVs in juveniles and adults. In relation to each behaviour, age and species, the authors provide a comprehensive and definitive review of pharmacological and genetic studies on the role of 5HT systems, concluding with some methodological comments aimed at improving study quality.

In the second paper, Servadio et al. present a similarly comprehensive and definitive review of the validity and utility of animal models of autism. This is a recent and burgeoning area of research, arising out of the growing concern over the apparent increase in the prevalence of autism spectrum disorders. The authors consider how the
DSM-V criteria of 'deficits in reciprocal social communication and social interaction', 'repetitive and stereotyped behaviors' and 'associated symptoms' can be operationalized in animal models, and examine how effectively these features of autism are implemented in the existing genetic (e.g. the BTBR and FMR1-knockout mouse strains) and environmental [e.g. prenatal exposure to valproic acid (VPA)] models.

Next, Blanco-Gandía et al. provide an encyclopaedic review of the effects of drugs of abuse on rodent social behaviour, focussing on three behavioural models: social play in adolescents, social interaction in adults, and the resident-intruder test as a model of aggressive behaviour. Within this framework, they systematically cover the effects of alcohol, cocaine, opioids, MDMA, cannabinoids, nicotine and other drugs of abuse, including prenatal effects on later behaviour and withdrawal from chronic drug administration. For each of these drug classes, the review effectively summarizes what is known of their effects in each of the three behavioural models, and the areas where results are controversial, with summary tables that we anticipate will be widely studied.

Essentially the same range of drugs of abuse is reviewed by Miller $e t$ al. in a different context: their effects on human responses to emotional facial expressions. Drugs are frequently consumed in social contexts, and their attractiveness may be modulated by effects on the processing of emotional responses in social settings: both increased reactivity to positive expressions and decreased reactivity to negative expressions may facilitate social interaction and thereby influence drug consumption. For each of the drugs considered, the authors review subjective, behavioural and physiological evidence of their acute and chronic effects on perceptual processes, and consider the potential role of effects on social perception in the development of addiction.

Starting from the premise that impaired social interaction is a hallmark of substance abuse disorders, and therefore that reorientation to social interaction may help to wean people away from drug use, Zernig and Penheiro review work from their laboratory in which experience of social interactions is used to counter the attractiveness of cocaine, as evaluated using place conditioning procedures in rats and mice. The social inhibition of cocaine preference was associated with inhibition of associated 
gene expression and electophysiological markers in dopamine-receptive GABAergic projection neurons of the medial nucleus accumbens. This intriguing line of work raises the possibility of a novel pharmacotherapeutic strategy to counter addiction by developing drug treatments that enhance social behaviour.

In the first of two reviews of drug effects on sexual behaviour, Angoa-Pérez and Kuhn consider the role of 5HT. Although 5HT has long been considered to inhibit sexual behaviour, recent pharmacological and genetic research, mainly in rodents, has identified that different 5HT receptor subtypes are associated with both inhibitory and facilitatory effects, often sexually dimorphic, on sexual arousal, motivation and behaviour. In addition to reviewing this literature, the paper includes a detailed summary of the neuroanatomical structures involved in male and female sexual behaviour. The second review, by Rodríguez-Manzo and Canseco-Alba, considers the role of endocannabinoids in male sexual behaviour. They point out that some effects of endocannabinoids are biphasic, with stimulation at low doses and inhibition at higher doses (as is the case, e.g. for effects on food intake), and they review the evidence for a similar biphasic effect on copulatory behaviour. The paper includes original research demonstrating that the lowdose stimulatory effect of anandamide on male sexual behaviour is mediated through $\mathrm{CB} 1$ receptors, whereas the high-dose inhibitory effect involves the activation of pain-related TRPV1 channels.

Finally, Li summarizes recent studies on the behavioural and neurobiological mechanisms of antipsychotic disruption of maternal behaviour. The evidence suggests that, at clinically relevant doses, antipsychotics disrupt maternal responses primarily by suppressing maternal motivation, although sedative effects of atypical neuroleptics also contribute, particularly to disruption of nursing behaviour. These effects of typical and atypical antipsychotics are mediated mainly through dopamine D2 receptors and 5HT2A/C receptors, respectively, with a predominant site of action in the nucleus accumbens shell. This understanding may lead to the development of strategies to help patients cope with the undesirable side-effects of antipsychotic medication.

\section{Part 2: Social modulation of drug effects}

Part 2 of this Special Issue contains papers reporting empirical studies in which the effects of drugs are influenced in a variety of ways by social context or prior social experience. The first paper, by Reymarova et al., considers the impact of social context on human smoking behaviour. The study found that nondependent smokers earning puffs on a cigarette by performing a progressive ratio task worked harder and smoked more when in the presence of another smoker. Similar effects of shared experience can be demonstrated in animals. Smith et al. report that rats treated with cocaine in the presence of another cocaine-treated rat subsequently showed a preference for a cocaine-treated rat over a saline-treated rat in a choice test. A paper by Watanabe reports a similar experiment in which methamphetamine-treated rats developed a place preference for the chamber associated with a methamphetamine-treated cagemate over that associated with a saline-treated cagemate. However, this effect was not seen with morphine. Together, these three papers suggest that common experience with psychostimulants (but not opiates) induces a social facilitation of their rewarding effects.

The next three papers address different aspects of social stress. In a study by Murgatroyd et al., lactating mothers were confronted daily by a male intruder, requiring the mother to defend her litter. This resulted in impaired maternal care and lactation, and increased anxiety, anhedonia and aggression, associated with changes in the mRNA expression of oxytocin, CRH, glucocorticoid receptors and orexin 2 receptors in different brain areas. Donahue $e t a l$. studied the effect of repeated defeat stress on $\kappa$-opioid receptors and their endogenous ligand, dynorphin, in male mice. Their evidence suggests that endogenous $\kappa$-opioid receptors on mesolimbic dopamine neurons are involved in the acute effects of social stress, which include anhedonia and social avoidance, but they play a more complex role in the resilience that develops in some animals following chronic social stress. In the third study, Baptista-de-Souza et al. show that mice cohabiting with cagemates undergoing neuropathic pain themselves showed an increase in pain sensitivity and anxiety. The mechanisms underlying this animal model of empathy are as yet unknown.

Social play, the most prominent form of social behaviour in young mammals, is thought to be important for social, emotional and cognitive development, and some studies have suggested that deprivation of social play may increase the risk for addictive behaviour. Lesscher $e t$ al. studied the long-term consequences of brief social isolation in rats during the peak period of social play. When tested as adults, isolated animals showed increased consumption of alcohol (albeit that operant responding for alcohol was unchanged, suggesting that motivation was not increased). The results support earlier suggestions that early social experience may protect against excessive alcohol use.

Two methodological papers follow, in which aspects of social behaviour are exploited for drug-screening purposes. Hasebe et al. have previously demonstrated that isolation-reared mice show hyperactivity during an encounter with an intruder. Here they report that encounter-induced hyperactivity was reduced by acute administration of a variety of antidepressants, and demonstrate a role for $\alpha 2$-adrenoceptors and 5HT4 receptors in these effects. However, as with other acute antidepressant-screening tests, some nonantidepressants 
were also effective. Schaefer $e t$ al. describe a new procedure for studying shoaling in adult zebrafish, which have recently emerged as a productive species for neurobiological and genetic investigations. In their procedure, single zebrafish are placed on either side of a glass partition. Compared with a single fish, the pair swim higher in the tank and closer to the central partition; benzodiazepines increased the separation of the pair of fish, whereas serotonergic antidepressants further increased their height in the tank. The authors suggest that this apparatus might be better than a tank containing multiple fish as a platform for low-cost high-volume screening of (water-soluble) potential anxiolytics and antidepressants.

In a further methodological study, Uphouse et al. describe the development of a procedure for studying antidepressant-induced female sexual dysfunction. This is a major side-effect of antidepressant medication, associated with poor medication compliance, but although animal models of female sexual motivation exist, they are insensitive to the effects of antidepressants. Here, female rats were trained to perform a nose-poke response that was reinforced by access to a sexually active male. In contrast to the lack of effect seen in other models that lack the social training component, this operant behaviour was decreased by a low dose of fluoxetine.

\section{Part 3: Drug effects on social behaviour}

In the third part of this Special Issue, we consider drug effects on a variety of social behaviours. The first paper, by Raza et al., examines the nature of the social dysfunction in one of the most widely used animal models of autism, prenatal exposure to VPA (cf. the review by Servadio et al. in the first part of this Special Issue). It was predicted that VPA-exposed rats would play less as juveniles. This was not found: the frequency of play was unaltered; however, when playing, the VPA-exposed animals were less likely to behave in ways that facilitated social contact from their playmate, and vocalized less, suggesting that VPA does produce deficits in juvenile behaviour that are consistent with those seen in autism. In another paper on juvenile play, Siviy et al. compared the effect of amphetamine in juvenile Sprague-Dawley rats with that in Fischer 344 rats, which are much less playful. The two strains differed in the effects of amphetamine on dopamine release from brain slices and on psychomotor activity, but were similar in the effect of amphetamine to decrease social play, supporting earlier conclusions that this is a noradrenergic rather than a dopaminergic effect. Another paper, by Sorensen et al., examines the social behaviour of a second animal model of autism, the FMR1 knockout mouse (again, cf. the review by Servadio et al. in Part 1). These animals, they found, were similar to control animals in the time spent with a stranger mouse, after correcting for the fact that they were hyperactive. However, the FMR1 knockout mice were unable to distinguish between a stranger and a familiar mouse, again supporting the presence of an autism-relevant social dysfunction.

Next follow three papers using social behaviours to examine psychotherapeutic effects relevant to schizophrenia. In the first of these, Cheng et al. used social recognition memory as a model of the cognitive impairments caused by reserpine, which nowadays is used primarily to control blood pressure but in days gone by was used as an antipsychotic agent. Reasoning that reserpineinduced monoamine release causes oxidative damage, they examined the effect of the antioxidant EGCG (the major psychoactive component of green tea). EGCG protected against both the oxidative and memoryimpairing effects of reserpine, suggesting that EGCG may have clinical potential to counter reserpine-induced cognitive impairments. In the second paper, Deiana $e t$ al. used the NMDA antagonist MK-801 to model negative symptoms of schizophrenia, and assessed the efficacy of several atypical antipsychotics with different pharmacological profiles as potential treatments. They found that MK-801 impaired social recognition memory, which aripiprazole dose-dependently prevented, but risperidone and olanzapine did not. Unexpectedly, cannabidiol, which has some antipsychotic activity in the clinic, did not reverse the effect of MK-801 and blocked the effect of aripiprazole. The authors suggest the 5HT1A receptor as a potential locus for this interaction. The third paper, by Holuj et al., has a similar rationale. Here, another NMDA antagonist, ketamine, was used to produce social withdrawal, which was reversed by the atypical antipsychotic amisulpride, but not by sulpiride or haloperidol. Further experiments suggested that the prosocial effect of amisulpride may involve antagonism of the $5 \mathrm{HT} 7$ receptor.

The next two papers examine aspects of the impairment of social interaction by psychostimulants (as reviewed, inter alia by Blanco-Gandía $e t$ al. in the first part of this Special Issue). Slamberova $e t$ al. compared the effects of amphetamine, cocaine and MDMA on social interaction in adult rats. All three drugs (including the 'party drug' MDMA) decreased social interaction in general, but specific patterns of social activity were differentially affected, with each drug having a unique profile of effects, which for amphetamine and cocaine, but not for MDMA, included active avoidance of the other animal. Janetsian et al. also saw a decrease in social interaction and increased avoidance following acute administration of methamphetamine. However, these effects were absent in animals displaying a sensitized locomotor response following a course of repeated methamphetamine administration that was previously shown to cause memory impairments. The authors suggest that the impairment of social interaction may reflect an acute anxiogenic effect. 
The final study examined the effect of acute administration of oxytocin on human aggressive responding. Alcorn $e t$ al. reasoned that, because oxytocin is known to have strong prosocial effects, it might decrease aggression in a laboratory procedure in which participants are led to believe that they are being aggressed against. Contrary to the hypothesis, not only did oxytocin not decrease aggression, but in the oxytocin group aggressive responding was positively correlated with psychometric measures of interpersonal manipulation and anger. It is not obvious why this occurred, but the results do highlight the need for a better understanding of individual differences in oxytocin effects. (Perhaps some illumination might be found in a Special Issue on The Behavioural Pharmacology of Oxytocin that will be published in Behavioural Pharmacology towards the end of 2016 - as described in the accompanying Announcement.)

\section{Commentary}

We were delighted by the enthusiastic response to our call for submissions to this Special Issue. We were pleasantly surprised by the volume of papers we received, but even more so by the quality and the diversity of the submitted work. The almost 30 articles published in these three issues of Behavioural Pharmacology show clearly that the pharmacology of social behaviour is a rich and blossoming research field. This work demonstrates how pharmacological interventions can be used to understand the neurobiology of functional, adaptive social interactions, how these interventions can be used to reveal alterations in social behaviour produced by psychoactive drugs, and how they can be used to understand how social functioning is impaired in psychiatric disorders such as autism and schizophrenia. All of these approaches feature strongly in this Special Issue, which also nicely illustrates the evolution and importance of social behaviour across the life span of humans and animals, ranging from social play through sexual and aggressive to parental behaviour. We are confident that the pharmacology of social behaviour has a bright and vibrant future, and it is with great pleasure and pride that we present these articles. We hope that you will enjoy them as much as we do.
Paul Willner Jack Bergman Louk Vanderschuren Bart Ellenbroek July, 2015 